\title{
Daily time management and autonomy in school-age children with ADHD, intellectual disabilities, and typically developing children show different patterns
}

Birgitta Wennberg ( $\nabla$ birgitta.wennberg@liu.se )

Linkoping University https://orcid.org/0000-0002-1752-8325

Anette Kjellberg Linköping University

Per Anders Gustafsson

Linköping University

\section{Lena Almqvist}

Mälardalen University

Gunnel Janeslätt

Centre for Clinical Research Dalarna

\section{Research article}

Keywords: ADHD, autonomy, child , cluster analysis, intellectual disability, participation, time management

Posted Date: October 7th, 2019

DOI: https://doi.org/10.21203/rs.2.11687/v2

License: (c) (i) This work is licensed under a Creative Commons Attribution 4.0 International License. Read Full License 


\section{Abstract}

Background Children with ADHD and those with intellectual disability (ID) often have problems in daily time management (DTM) and the underlying time-processing ability (TPA). It is, however, less well-known how DTM and TPA may impact children's everyday autonomy. The purpose of this study was to investigate DTM, TPA and selfrated autonomy in the activities of everyday life among children aged 9-15 years with ADHD ( $n=47)$, with ID ( $n=$ $47)$ and typically developing (TD) children $(n=47)$. Method A descriptive, comparative and cross-sectional design was used. Group comparisons and cluster analysis with one-way analysis of variance (ANOVA) with Tukey post-hoc tests and bootstrapping were used to analyse the data. Results Children with ADHD and those with ID had significantly lower TPA and DTM than TD children. However, there was a considerable heterogeneity among the children with ADHD and ID ranging from skilled to having significant problems in TPA Children with ADHD had lower DTM than those with ID. For all children the levels of self-rated autonomy seemed to follow the level of TPA. Conclusions Children with ADHD and those with ID have an increased risk of delayed TPA, affecting their DTM and autonomy. The results indicate a need to measure TPA and tailor interventions for each child.

\section{Background}

\section{Daily time management and time-processing ability}

Managing one's time (Daily time management, DTM) is important in order to perform everyday activities and to become autonomous and independent (1-3). DTM is partially based on a person's cognitive functioning related to time (2, 4-6); namely, a person's TPA (2). TPA develops during childhood and adolescence and seems to follow the developmental stages, as indicated by chronological age in children without disabilities $(3,7)$. TPA is an unidimential construct containing three categories: time perception, time orientation (divided into two sub-levels: time concepts and objective time) and time management which can be seen as different levels of complexity of TPA (8). Time perception (experience of time and durations of activities) and time orientation (i.e. to tell time and to know what day and month it is) are basic levels of TPA and are necessary for time management $(2,6)$. Time management, defined as ordering events in chronological sequences and allocating the appropriate amount of time for activities, is an important part of executive functions $(5,6)$. Executive function refers to brain functions that activate, organize, integrate and manage other functions $(9,10)$. It enables individuals to account for short- and long-term consequences of their actions and to plan for the sequent results. It also allows individuals to make realtime evaluations of their actions and make necessary adjustments if those actions are not achieving the desired result. The concept of executive functioning could be regarded as an underlying capacity for autonomy and for the sub-concept time management within the cognitive function of time-processing ability (TPA). Transferring the cognitive functions into managing one's time in daily life (DTM) is a challenge and takes a lot of practice. According to the International Classification of Functioning, Disability and Health (ICF) contextual factors as environmental factors and personal factors can hinder or facilitate a person's functioning (5). (See Fig. 1).

In a study of young children aged 5-10 years, with and without disabilities, the results showed four typical patterns related to the chronological age of the children without disabilities (7). There were children with and without disabilities in all patterns, but children with a diagnosis were significantly older in all groups except for the group with the most skilled children. Parents' rating of their child's DTM was related to the child's TPA. The more skilled the child was in TPA, the higher the parent rated the child's DTM.

\section{Cognitive impairment, autonomy and participation}


Cognitive impairment affecting TPA is not always visible, but can still lead to reduced autonomy and participation in everyday life. According to Cardol, De Jong \& Ward (11), personal autonomy can have two dimensions: deciding what to do (decisional autonomy) and doing what one has decided (executional autonomy). Decisional autonomy loads heavily on cognitive functions, i.e. to know one's capacity and preferences, to know the demands of the activity, to take into account pros and cons, and finally to decide what to do and when to do it (12). Executional autonomy, according to Peny-Dahlstand et al. (13), is likewise dependent on the ability to initiate, organise, adapt and proceed with the task until the goal is reached), i.e. observable executive functions. In their study of children with spina bifida (6-14 years old), children's levels of autonomy were strongly related to the performance of everyday activities, and in particular to activities depending on executive functions. Rosenberg (14) also states that executive functions are vital for young children's independence and autonomy in daily life. Also in Janeslätt et al. (7) the children's self-rating of autonomy was related to their TPA, except for the youngest children (5 years).

According to the ICF participation is defined as "involvement in a life situation" (5). Autonomy is suggested to be a prerequisite for an individual's ability to participate in his or her current environment $(11,15)$ and high self-ratings of autonomy have been shown to be related to a high degree of self-rated participation $(16,17)$ and good quality of life (18). Personal characteristics such as autonomy and internal locus of control have been related to participation at school for children with disabilities (16).

\section{Children with ADHD and difficulties with time}

Several researchers have emphasised deficiencies in the executive functions as a basic problem for children with $\operatorname{ADHD}(9,19)$. Children with ADHD have been shown to have a delayed maturation of the cerebral prefrontal regions, which are important for executive functions, including time management $(20,21)$. Children with ADHD also seem to have a different sense of time than typically developing children (22). Their ability to discriminate and reproduce time intervals and to make retrospective time estimations has been shown to be impaired (23-25). The perception of time is mainly researched in experimental studies $(24,26,27)$. Mioni et al. (28) found that children with ADHD performed less precisely in time-based prospective memory tasks and that time perception predicted prospective memory accuracy. Children with ADHD may also have reduced ability to value time in relation to the actual duration of daily activities (7). These difficulties are apparent mainly in the experience of time, and planning and organising the plan when carrying out daily activities, at home, at school and during play (29). In a study of Lindblad and colleagues (30) children with ADHD 6-16 years had lower adaptive functioning compared to children with mild intellectual disabilities (ID).

\section{Children with ID and difficulties with time}

Children with ID have a cognitive development, including the development of TPA, that is slower than in children without disabilities, and the level of abstract thinking as an adult is lower (31). It is well known that difficulties with abstract concepts such as the understanding of time concepts $(31,32)$ and a reduced working memory $(31)$ are common in children and adults with ID. This affects the practical skills in activities in daily life (33). Clinically, children with ID have been targeted with interventions directed towards time deficits for many years, but nevertheless there are few studies on TPA and DTM (32). In one of the few studies involving children with ID, the children made more errors in prospective memory tasks than children without disabilities (34). They also had a weaker time reproduction than children without disabilities. It has been shown that children and adults with ID benefit from time-assistive devices to enhance DTM and TPA (35-38) and that they can improve their TPA by training in low levels of TPA that focuses on time perception and the duration of activities (39). The fact that 
children with ID experience limited participation and autonomy in school activities has been presented in previous studies $(17,40)$.

\section{Self-rated autonomy in children with ADHD and children with ID}

Autonomy in children with disabilities is mostly studied in a school context. Results show lower participation and self-rated autonomy in children with disabilities compared to children without disabilities $(17,40)$. In Eriksson et al. (40), 66 children aged $7-12$ years with and without disabilities were observed at school for one day and were also interviewed about participation and asked to self-rate their autonomy. The results showed that children with disabilities, mostly children with ID, ADHD and children with motor impairment, had lower participation and selfrated autonomy compared to children without disabilities. In Eriksson \& Granlund (17), a comparison between 959 children with and without disabilities in the ages 7 to 17 years, children with disabilities, including children with ID, rated their participation and autonomy in school- activities lower than children without disabilities. Studies in children with ADHD and children with ID self-rating their autonomy outside school context are sparse. We only found two studies with children self-rating their autonomy in everyday activities. In Janeslätt et al. (2) 118 children with different diagnoses, including ADHD and ID, aged 6-11 years, self-rated their autonomy. Also parent ratings of DTM and measurements of TPA were conducted. Results showed a significant relation between self-ratings of autonomy, parents' ratings of DTM and the children's TPA. The same pattern was shown in Janeslätt et al (7) were children with $(n=77)$ and without $(n=89)$ diagnosis and aged 5-10 years self-rated their autonomy. As in the previous study, autonomy and DTM were related to the children's TPA. Children with disabilities were found in different clusters, showing diversity in TPA, DTM and autonomy not explained by diagnosis.

Previous studies on TPA in children with ADHD and children with ID have especially focused on the first sub-level of TPA, time perception $(22-26,34,41))$, and a few studies on the sub-level of time management $(34)$. All were laboratory studies using standardised tests. We found only two studies investigating the whole construct of TPA including time perception, time orientation and time management and using more practical tasks reflecting real-life activities $(2,7)$. However, in these studies the children were younger (age 5-10 years and 6-11 years) and also children with other diagnoses than ADHD and ID participated. Also studies on self-rated autonomy in children with ADHD and children with ID outside school-context are sparse (42). Despite indications of differences in TPA, DTM and autonomy not explained by age or diagnosis, reported in the study by Janeslätt et al. (7) there is, as far as we know, no study investigating and comparing patterns of TPA, DTM and autonomy in children aged 9-15 with ADHD, ID and typically developing children (TD). There probably are different dimensions of TPA in children that develop individually depending on age, diagnosis, gender, etc. Also it is possible that such development differs in children with functional variations compared to TD children. To investigate such patterns could give important knowledge on which interventions could be individualised.

The aim of this study is to investigate DTM, TPA and self-rated autonomy in everyday activities in children aged 915 years with ADHD, compared to children with ID and to TD children.

Research questions:

1. Are there any differences, and if so what, in TPA, DTM, and self-rated autonomy in everyday activities between children with ADHD, children with ID and TD children?

2. Can different patterns of TPA, based on the different levels of TPA, be identified in children with ADHD, children with ID and TD children? 
3. Do children within the same diagnostic category or within the same age span share membership of a group with a specific pattern of TPA?

4. Do the parent-rated DTM and the self-rated autonomy of children differ between patterns?

\section{Method}

A descriptive, comparative and cross-sectional design (43) was used. Children with ADHD, where the concepts of DTM/TPA/autonomy have recently been introduced, were compared with children with ID where difficulties in all aspects of daily functioning including DTM/TPA/autonomy are expected, and typically developing children.

\section{Participants}

\section{Children with ADHD}

A consecutive sampling was used in the study and the children with ADHD were recruited from three child and adolescent psychiatric clinics (CAPs) and one children's habilitation service (HAB) in Sweden between September 2012 and Mars 2015. Inclusion criteria were a diagnosis of ADHD determined in accordance with DSM-IV criteria by an experienced CAP specialist after a thorough neuropsychological investigation encompassing careful clinical examination and questionnaires, a steady prescription of ADHD medication for at least three months, age 9-15 years and parent-reported difficulties with DTM, and ten points or more on a clinical rating of 15 statements related to problems with DTM (a subscale of the Five to Fifteen parent questionnaire (44). Examples of statements were if the child was stressed by time limits, had difficulties in using a watch or if the child was "lost in his/her own world" and was not aware of the passage of time. Further examples were when a child had obvious difficulties in carrying out and completing morning routines and arriving in time for school or had difficulties in calculating the time span for daily activities and leisure activities (for more information see 45). Exclusion criteria were ID (IQ<70, autism spectrum disorder, or language barriers (e.g. not being able to answer questionnaires in Swedish). This resulted in 47 children with ADHD. Parents of the children were asked to participate in the study during ordinary monitoring visits to CAP or HAB. Those who agreed to participate were given written invitations to a personal visit for the data collection. Data collection was conducted with the child and the parent separately (see 45 for more details).

\section{Children with ID and TD children}

To enable comparisons each child with ADHD was matched, pairwise, with a child with ID ( $n=47)$ and a TD child ( $n=$ 47) based on sex and chronological age. Children in the comparison groups were samples drawn from a database holding data collected between 2006 and 2015 containing data from children with different diagnoses and from TD children. Data was gathered by certified occupational therapists working within habilitation services and participating in courses for KaTid (see below) certification. The data from the comparison group of ID children were all from children within the ordinary work area of the occupational therapists. All children were diagnosed having mild or moderate ID and three of them with comorbidity (autism spectrum disorder), cerebral palsy and impairment of hearing and language impairment). None of the children with ID were diagnosed with ADHD.

TD children were recruited as a convenience sample by the participants in courses for KaTid certification from the normal population, self-reported by the parents being healthy and having no diagnosis, and thereafter included in the database. In total there were 141 participants.

\section{Procedure}


The children's TPA was measured using a structured assessment (KaTid), and the children's DTM was rated using a parent questionnaire (Time-Parent scale). The children's autonomy was rated by self-reporting (self-report Autonomy scale).

\section{Instruments}

Kit for assessing time-processing ability (KaTid) (6) is an instrument for the assessment of time perception, time orientation (divided into the subscales time concepts and objective time) and time management for children with an age up to10 years (KaTid-Child version) and adolescents with an age from about 10 years (KaTid-Youth version). It is a table-top test to be performed by a trained certified professional, e.g. an occupational therapist. The child responds to questions and performs practical exercises, such as setting a timer for a fixed time, and placing picture sequences in the correct order. KaTid-Child contains 57 items measuring: time perception (15 items), time orientation (32 items) and time management (10 items), summarised into one measure of TPA. KaTid-Youth contains the same categories, but consists of 59 items, 33 of which are the same as in KaTid-Child. KaTid-Child and KaTid-Youth have been shown to have good validity and reliability with children and adolescents, with and without disabilities, including children with ADHD and children with ID, in measuring change $(36,39,45)$. The two versions of KaTid measures the same construct, TPA (6). An earlier cluster analysis indicated that the patterns of TPA mainly follow the chronological age of children without disabilities, all clusters differing as regards to level of TPA (7). The level of TPA seems to be a more valid overall base than the type of diagnosis for the planning of interventions in DTM (7). KaTid-Child has been validated and tested for internal consistency in children with and without disabilities, with Cronbach Alphas of 0.78-0.86 $(2,8)$. KaTid have been used with TD children, for children with ADHD, Autism, mild or moderate level ID, Spina bifida (SB), Cerebral Palsy (CP), and double diagnosis including at least one or more of the diagnoses above. In this study, KaTid-Child or KaTid-Youth was used, depending on the age of the child. However, for three of the older children with ID KaTid-Child was used because the KaTid-Youth version was considered too difficult. The certified and clinical experienced occupational therapists estimated that KaTidYouth could be used for all TD children regardless of age.

In the Time-Parent scale, the parents rate their children's DTM: understanding concepts of time, managing time and adapting to time demands $(2,8)$. There are two versions: children with an age of $5-10$ years and adolescents with an age of 10-17 years. Both questionnaires consist of 12 statements rated on a Likert frequent/agreement scale with four agreement response options scored from never/I don't agree at all (1p) to always/I completely agree (4p). There is also an option: do not know (0p). The statements in the two versions capture the same ability but are set to different degrees of difficulty and are customised to the different age ranges. The Time-Parent scale has been validated and tested for internal consistency in children and adolescents with and without disabilities with Cronbach Alphas of $0.79-0.86$. It has been found to be psychometrically sound and significantly correlated with TPA as measured by KaTid $(2,8)$ and can measure change $(45)$.

Using the Autonomy scale of the instrument "Children's participation in school, students' assessment forms" (16, 17), the children rated their autonomy. This scale is an adapted short version of the Autonomous Functioning Checklist Self-determination scale (46), where the autonomy items are based on Sigafoos et al. (47). There are two versions: for children aged 7-12 years and for adolescents aged 13-17 years. Children rate their autonomy in everyday activities or situations that they usually do or decide upon by themselves, based on 23 statements (children's version) and 30 statements (adolescent's version). The items are divided into sections: daily routines, interacting with people, recreational and leisure choices, community involvement, and personal style. In the adolescents' version there is an additional section: education and planning for my working life. It has a Likert 
response scale for frequency: I do not do it (1p), sometimes (2p), often (3p) and always (4p). The Autonomy scale has been validated and tested for internal consistency with a Cronbach Alpha of 0.82 for children aged 7-12 years and 0.93 for adolescents aged 13-17 years (16). There is also a short version, Autonomy Short, for children who need an even shorter questionnaire. It is based on 14 items with the same four response alternatives as the other versions. All seven items capturing autonomy in daily routines from the original children's version are included, as well as some items concerning choices and interacting with people. Autonomy Short has been shown to be psychometrically sound in construct validity (8) and it has been demonstrated that a version using the first 12 items can be used to measure self-rated autonomy equally as well as the longer versions (7). In this study, all three versions were used. If needed, the child was offered support from the occupational therapist by reading or explaining the statements. The response scale was also offered as cognitive adapted with pictures.

\section{Ethical aspects}

The ethical guidelines stipulated by the Helsinki Declaration (48) guided the research process. Ethical approval for this study was granted by the Regional Ethical Review Board in Linköping for the children with ADHD (D-nr. 2012/166-31) and for the children with ID and the TD children (D-nr. 2005/032).

Written informed consent was obtained from all parents and from the older children/adolescents in the study.

\section{Data analysis}

All data analysis was performed using IBM SPSS Statistics version 26, selecting a significant level of .05 and a confidence interval of $95 \%$.

\section{Comparisons between groups}

Variable-based analysis was used to compare TPA, DTM and self-rated autonomy between children with ADHD, ID and TD children. To make it possible to compare the data for all children, some transformations of the different scales were needed (49). Data collected with KaTid-Child ( $n=37)$ were transformed into KaTid-Youth scores using a transformation scale, making the score sums comparable (6). A calculation to percentage of maximum scores was also made. Regarding Time-Parent scale there were two versions used. The sum of scores of the version of the Time-parent scale used for each child was transformed into a percentage of the maximum score. Three different versions of the autonomy scales were used. In order to compare autonomy for as many children as possible, the Autonomy Short and the version for 7-12-year-olds were combined into one (hereafter named "Autonomy-short"), including only those items that were identical in both versions. An analysis was conducted on the sum scores from the Autonomy-short version and the version for 13-17-year-olds respectively, and on the percentage of maximum scores from both versions.

Group comparisons were made using one-way analysis of variance (ANOVA) with Tukey post-hoc tests for multiple comparisons (50). A bootstrapping procedure with 1000 replicated Cls were conducted to correct for subgroup heterogeneity and small sample size. Reported $p$-values are adjusted accordingly Write et al. (50). In between analysis of Effect size (ES) for the different groups was calculated using Cohen's $d$ with ES $d=0.2-0.5$ representing a small effect, $d=0.5-0.8$ medium and $d>0.8$ large effect (51).

\section{Cluster analysis}

A hierarchical cluster analysis of patterns of TPA for children with ADHD, ID and TD children was conducted using SLEIPNER 2.0 (52) with Ward's method of agglomeration. Data used in the cluster analysis were scores from the 
four subscales of KaTid-Child and KaTid-Youth. Based on the fact that different versions of KaTid with different sum scores on each subscale were used for the children, it was necessary to convert to a percentage of maximal sum score for each subscale in the two versions of KaTid. An argument for this choice is the fact that there is no transformation scale (as was used in the comparison analysis) for the subscales. Also, it is the patterns of TPA being investigated in this second analysis rather than the actual difficulty of the subscale. In this study, the following criteria were used to identify the number of clusters: (i) a homogeneity coefficient of at most 1.00 (preferably <0.50), (ii) a percent of the total explained error sums (ESS) of at least $67 \%$ and (iii) a distinctive break in the scree plot indicating a large increase in ESS and the number of clusters to retain before the increase and, (iiii) split sample replicability through a total rerun of the cluster analysis on a random half of the sample of at least 0.30 , the mean in ASED between the clusters in the two solutions $(52,53)$ using the Random module. Three random samples were run $(n=70)$, resulting in one cluster solution with five clusters and two solutions with four clusters. The first cluster solution included two residue cases, while the other two did not include any residue cases. After removal of the two residue cases in the first cluster solution, a reliability analysis was run of the random split samples, demonstrating an average pairwise matching between the clusters of 0.34 . Eventually, a four-cluster solution was chosen, as it appeared to best match the data according to the above criteria. These statistical criteria's provided a rough guidance regarding the number of clusters to retain, however, also a theoretical examination of the cluster solution guided the decision. The resulting four-cluster solution matched the theoretical underpinnings of the study and seemed meaningful in relation to earlier findings $(2,7)$ of TPA of children with and without disabilities.

Comparisons were made concerning differences in age, TPA, DTM and autonomy, and between clusters, using oneway analysis of variance (ANOVA) and bootstrapping (see above). Chi-square tests were used to analyse differences between the clusters regarding sex and diagnosis (54). To determine whether there were differences within the clusters, sub-group analyses were also performed regarding age and sex in the two clusters with the largest numbers of participants.

\section{Results}

Mean age of the children with ADHD was $11.5 \mathrm{yr}$. (SD 1.93), children with ID $11.6 \mathrm{yr}$. (SD 1.83) and for the TD children $11.5 \mathrm{yr}$. (SD1.90). Most of the children were boys $(n=32,68 \%)$.

\section{Missing data}

There was no missing data in TPA and DTM. In self-rated autonomy, there was missing data from one child with ADHD and from nine children with ID. A sensitive analysis did not show any significant differences using the whole sample compared to only using data from children with no missing data.

\section{Differences in TPA, DTM and autonomy between groups}

\section{Time-processing ability}

There were statistically significant differences between the groups, $\mathrm{F}(2,138)=84.49, p<.001$. TD children had the highest scores for TPA, measured by KaTid and all data converted to KaTid-Youth, with a mean of 51.6 (SD 8.4), children with ADHD the second highest scores for TPA (mean 42.4, SD 11.0) and children with ID the lowest scores for TPA (mean 23.2, SD 12.6) (Table 1). There were statistically significant differences between children with ADHD and TD children $(p<.001)$, children with ADHD and children with ID $(p<.001)$ and children with ID and TD children $(p<.001)$ with large ES on all comparisons (Table 1$)$. 


\section{Daily time management}

Statistically significant differences in daily time management were found between the groups, $\mathrm{F}(2,138)=27.45, p<$ .001. TD children had the highest scores for DTM (mean 66.2, SD13.8) according to percent of maximum score of the Time-Parent scale (Table 1), children with ID had the second highest scores for DTM (mean 57.1, SD 17.1) and children with ADHD had the lowest scores for DTM (mean 44.6, SD 10.2). There were statistically significant differences between children with ADHD and TD children $(p<.001)$, between children with ADHD and children with ID $(p<.001$ and between TD children and children with ID $(p=.007)$. The ES were large or medium (Table 1$)$.

\section{Autonomy}

There were statistically significant differences between the groups in autonomy, $\mathrm{F}(2,128) 30.58, p<.001$. TD children had the highest scores in both autonomy versions, followed by children with ADHD (Table 1). Children with ID had the lowest scores in both autonomy scale versions.

Using percent of maximum score based on both versions of autonomy TD children had the highest scores (mean 80.7, SD 10.4), children with ADHD the second highest scores (mean 76.0, SD 9.1) and children with ID had the lowest scores $(61.3, S D 15.3)$. There were statistically significant differences between children with ADHD and children with ID $(p<0.001)$ and between TD children and children with ID $(p<0.001)$ with large ES.

\section{Patterns of TPA in children with ADHD, children with ID and the TD children}

The cluster analysis resulted in four clusters with different patterns of TPA, all based on the four subscales of TPA. The evaluation of the explained error sums of squares (69.70\%) demonstrated an acceptable value. The homogeneity coefficient of the groups differed between 0.451 (Cluster C), 0.706 (Cluster B), 0.709 (Cluster A) and 1.131 (Cluster D), which is acceptable (Table 2).

\section{Outliers}

The first step in the cluster analysis was to identify a residue of outliers. The participants in the residue are held as being dissimilar to all other participants. There were two residuals found, both ID children. In total, 139 participants were included in the clusters.

\section{Descriptives}

There were statistically significant differences between clusters in age $F(3.135)=3.25, p=.024$. The distribution of sex in the clusters varies between $57 \%$ and $86 \%$ boys. Still, there were no statistically significant differences in sex between the clusters $(p=.756)$. There were statistically significant differences between clusters according to diagnosis $(p<.001)$. Children with ADHD were represented in three of the four clusters and TD children in two. There were children with ID in all clusters. All clusters show the highest proportion of correct answers at the lower levels of TPA, i.e. time perception and time orientation/concepts, and a reduced ability at the higher levels, i.e. time orientation/objective time and time management (Figure 2). There were statistically significant differences in TPA between clusters $F(3,135)=185.40, p<.001$.

Descriptions of the participants in each cluster according to number, sex, diagnosis, mean age, TPA (total score, time perception, time orientation (concepts and objective time) and time management). DTM and self-rated autonomy are presented in Table 2. The results for each cluster are presented separately below. 


\section{Cluster A - (Skilled in all levels of TPA)}

Cluster $A$ is the largest $(n=79)$ and contains children from all groups, but children with ID are underrepresented (11\%). TD children make up the largest proportion (47\%) followed by children with ADHD (42\%). Mean age of the children is 11.8 (SD 1.92; range 8.72-15.73), i.e. the oldest children except for Cluster D (containing only children with ID). TD children in this cluster are the oldest of the TD children in the sample. Subgroup analysis of age in Cluster A showed that TD children had a mean age of 12.0 years, children with ADHD 11.7 years and children with ID 11.5 years.

According to TPA, these children have a mean of $82 \%$ of total scores, which is the highest in the sample. Children in this cluster are skilled in all levels of TPA: time perception, time orientation (concepts and objective time) and time management (Figure 2).

\section{Cluster B - (Skilled in time perception and time orientation/concepts)}

Cluster B is the second largest $(n=46)$ and contains children from all groups, children with ID being overrepresented (52\%). The distribution of TD children and children with ADHD is relatively even ( $26 \%$ and $22 \%$ respectively). Mean age of the children is 10.9 (SD1.66; range 8.62-15.14), which means that it is the cluster with the youngest children. The TD children in this cluster are the youngest of the TD children in the sample. Subgroup analysis of age in Cluster B shows a mean age for TD children of 9.8, children with ADHD 11.2 and children with ID 11.2.

According to TPA, children in this cluster have a mean score which is the second highest, $58 \%$ of total scores. Children in this cluster are skilled in time perception and time orientation/concepts but have difficulties with time orientation/objective time and time management (Figure 2).

\section{Cluster C - (Skilled only in time perception)}

Cluster $\mathrm{C}$ is small $(n=7)$, and contains children with ADHD and children with ID, children with ID being overrepresented (71\%). Mean age is 11.1 (SD 1.42; range 9.44-13.50). Children with ID are older $(M=11.6)$ compared to children with $\operatorname{ADHD}(M=9.7)$.

According to TPA, these children have a mean of $39 \%$ of total scoresi.e. the second lowest in the sample. Children in this cluster are skilled only in time perception and have difficulties with time orientation (both concepts and objective time) and time management (Figure 2).

\section{Cluster D - (Difficulties at all levels of TPA)}

Cluster $\mathrm{D}$ is small $(n=7)$, and contains only children with ID. Children in this cluster are the oldest in the sample with a mean age of 12.5 (SD2.24; range 9.66-15.58).

According to TPA, these children have a mean of $24 \%$ of total scores, which is the lowest in the sample. The children have difficulties at all levels of TPA but mostly with time orientation/objective time and time management (Figure 2).

\section{Diagnostic category and age related to pattern of TPA}

Children with ADHD are represented in three clusters, i.e. not in cluster D (Difficulties at all levels of TPA). Most children with ADHD are found in Cluster A (Skilled in all levels of TPA), in which they are about as numerous as the 
TD children, and in Cluster B (Skilled in time perception and time orientation/concepts).

Children with ID are represented in all clusters, with the largest numbers found in Cluster B (Skilled in time perception and in time orientation/concepts), where they are overrepresented. They are also overrepresented in Cluster C, containing children skilled in time perception but having difficulties with all other levels of TPA and in Cluster D (Difficulties at all levels of TPA), containing children with the lowest scores of TPA.

The TD children are represented in two clusters, Cluster A (Skilled in all levels of TPA) (79\% of TD children) and Cluster B (Skilled in time perception and time orientation/concepts).

Children in Cluster B (Skilled in time perception and time orientation/concepts) had the lowest mean age (10.9), followed by Cluster C (Skilled only in time perception) (11.1) and Cluster A (Skilled in all levels of TPA) (11.8). Children in Cluster D (Difficulties at all levels of TPA) had the highest mean age (12.5). The age range differs between clusters; Cluster A having the largest range (8.72-15.73) and Cluster C the smallest (9.44-13.50).

\section{Daily time management and autonomy}

DTM, as rated by the parents, is almost the same in all clusters, the exception being children in Cluster D (Difficulties at all levels of TPA), which has the lowest score ( $40 \%$ of maximum score). There were no statistically significant differences between the clusters.

There were significant differences in autonomy, as rated by the children themselves, between the clusters, $F(3,125)$ $=15.98, p<.001$. Children in Cluster A (Skilled in all levels of TPA) have the highest autonomy scores (78\% of maximum score), followed by children in Cluster B (Skilled in time perception and time orientation/concepts) (71\%), Cluster C (Skilled only in time perception) (60\%) and the lowest scores in Cluster D (Difficulties at all levels of TPA), with $42 \%$ of maximum score. There were statistically significant differences between Cluster $A$ and $B(p=.003)$, Cluster A and C $(p=.001)$, Cluster A and D $(p<0.001)$ and Cluster B and D $(p<.001)$.

\section{Discussion}

\section{Differences in TPA, DTM and self-rated autonomy in groups based on diagnosis}

Children with ADHD had statistically significant lower scores on TPA and DTM compared to TD children. Parents of children with ADHD rated their children's DTM as low, even lower than that of children with ID. These results indicate that children with ADHD may have both deficits in cognitive functioning regarding TPA and problems in daily activities related to time. This is in line with experimental studies showing deficits in time perception (24-26), temporal foresight (26) and time-based prospective memory tasks (28), together with intervention studies showing problems in organising and planning school activities $(29,55)$, daily activities at home $(45)$, and overall adaptive functioning (30) among children with ADHD.

Given that children with ID had the lowest TPA in the sample, we might expect the parents of children with ID to rate their children's DTM as lowest, but they did not. One explanation could be that parents of children with ID have low expectations of their child's DTM because of their known limitations in intellectual and adaptive functioning that are the criteria for the diagnosis (33). In addition it is clinically known that children with ID often have difficulties related to time (32). Another potential explanation may be that the children with ID might have had access to timeassistive devices, to compensate for deficits in TPA. Since in Scandinavia persons with ID are the target group who 
have had access to time-assistive devices for the longest, especially low-tech products such as adapted weekly schedules and/or activity schedules (36, 56-58).

Children with ADHD had a lower mean score for autonomy than TD children. Janeslätt et al. (7) showed in a study of children aged 5-10 years, with and without a diagnosis, that a child's self-rated autonomy was related to their TPA. The higher TPA a child had, the higher they self-rated their autonomy. Peny-Dahlstrand and colleagues (13) also found that high observed executive functions were related to high levels of self-rated autonomy in children with spina bifida. That both decisional and executive autonomy are dependent on cognitive functions has been found in a number of studies (11-13). The fact that children with ID in the present study, who had the lowest scores for TPA, also had the lowest scores for self-rated autonomy is in agreement with this. The lower levels of TPA in children with ADHD and ID in our study, compared to TD children, could therefore be an explanation for the lower scores on selfrated autonomy compared to the TD children. The fact that autonomy is suggested to be a prerequisite for participation $(11,15)$ highlights the importance of interventions supporting cognitive functions, including TPA, in children with ADHD and ID in order to enhance their autonomy and thereby support their participation.

\section{Patterns of TPA in children with ADHD, children with ID and TD children}

However, the differences between diagnoses groups described above do not take into account the diversity within the children with the same diagnosis. Therefore we went further with this first study of TPA with a person-oriented approach, based on all four subscales/dimensions of time-processing ability (see Figure 1) in children with and without a diagnosis, and it revealed four different clusters of TPA. All the clusters showed a similar overall pattern, with the highest scores being for time perception, and a decreased ability one by one for the subsequent higher levels of TPA. This is an indication of different dimensions of TPA in children, and investigating such patterns could give important knowledge to plan and implement individualised efforts to strengthen TPAs, based on the dimensions in which children lag behind in groups of children with similar patterns, rather than general (diagnose based) groups that vary widely in TPA. Our findings are in line with previous studies showing a development of TPA for TD children; starting with perception of time, followed by time orientation and later on time management $(3,8)$. The same pattern was found in a study including younger children, aged 5-10 years, with and without disabilities (7). An interesting finding was that in the present study all clusters showed a distinct difference in decrease between the subscales time orientation/concepts and time orientation/objective time. This difference was more evident in the clusters with lower overall levels of TPA. This indicates that there are sublevels within time orientation; that time concepts are easier to manage than understanding what objective time is. This is in line with Janeslätt et al. (7), who raised the issue of time orientation as two different strands. Based on this we suggest further research with larger samples.

\section{Specific patterns of TPA depending on diagnosis and age}

In the present study, there were statistically significant differences between clusters concerning diagnosis. Nevertheless, there are no signs of differences in the overall pattern of TPA, indicating that children with ADHD and ID have the same pattern of TPA development compared to TD children but for some of these children at a slower pace. The same was found in Janeslätt et al.,(7) were children with disabilities were older than TD children in the same cluster. This indicates a need for targeted and early intervention to enhance development of TPA (7). Training of organizational, time management and planning skills (OTMP) is a well-established treatment in children and adolescents with ADHD (59) and interventions focusing on TPA as a complement are therefore needed. Otherwise, the functional impact (of having ADHD or ID) may increase with age (60). 
Children with ADHD were represented in three of the four clusters, further emphasising the diversity of TPA among children with this diagnosis. This is in contrast to the TD children who, not surprisingly, were only represented in the clusters with the most able children. The fact that children with ADHD were more than a year older than TD children in Cluster B (Skilled in time perception and time orientation/concepts) and the presence of children with ADHD in Cluster C (Skilled in only time perception) indicates a delayed TPA for some children with ADHD, and some aspects of TPA, compared to TD children. This is in accordance with studies showing a delay in maturation in the prefrontal cortical regions important for executive functions, including time management, in children with ADHD $(20,21)$.

Children with ID were present in all four clusters, but overrepresented or the only one in the two clusters with children with the lowest TPA. They were also, like the children with ADHD, more than a year older than TD children in the Cluster B (Skilled in time perception and time orientation/concepts) and the only one in the cluster with the lowest TPA which contained the oldest children in the sample. Children in this cluster probably have a disability that affects most situations in everyday life; at home, at school and during leisure time. Still it is noteworthy that some children with ID are found in Cluster A (Skilled in all aspects of TPA). Taken together, children with ID in this study shows a great variety in TPA, not explained by age, indicating a need for individual measuring and tailored interventions for children with ID. A recent RCT study by Janeslätt et al. (39) showed promising results for 10-17-year-old children with ID that significantly increased their TPA, in only eight weeks, with a new method for training low levels of TPA.

\section{Patterns of daily time management and self-rated autonomy}

Except for Cluster D (Difficulties at all levels of TPA), there were almost no differences in parent-rated DTM between clusters, although the clusters differed in TPA. This result conflicts with a previous study showing that DTM is related to a child's TPA (7), however, these children were younger (aged 5- 10 years), and the diversity of children's diagnoses was larger including children with Autism spectrum disorder $(n=14)$, Spina Bifida and Cerebral Palsy $(n=20)$, mild or moderate level intellectual disability $(n=20)$ children with ADHD $(n=13)$ and typically developing children ( $n=89$ ). These differences between the present study and Janeslätt et al. (7) are probably the reason for the disparity between the results. As TPA is the cognitive functioning and DTM is the daily functioning in the aspects of managing time there could also be other explanations. According to Janeslätt et al. (2) parents' ratings of their children's DTM explained $25 \%$ of the variation in TPA. Other explanations could be for example environmental factors including support for understanding time provided by time-assistive devices, or support from parents and teachers $((2,5,7)$.

Interestingly, in the present study, there are contradictory results concerning DTM. Analysis of differences between TD children, children with ADHD and children with ID resulted in statistically significant differences in DTM but this can't be seen in the comparisons of clusters. This leads to a need for further research on TPA and DTM in children with ADHD and ID and with larger samples.

In contrast to DTM, there were differences in the clusters concerning self-rated autonomy. The patterns of autonomy seemed to follow the level of TPA. Clusters with high scores of TPA also have higher scores of autonomy. This is in line with the results in Janeslätt et al. (7) concerning children aged 5-10 years with and without a diagnosis.

\section{Methodological issues/limitations}

A strength of the study is that two different methods were used for analysing the data: a group comparison (43) to answer the broader question of TPA in children with functional variations compared to TD children, and a personoriented cluster analysis (53) to look into dimensional aspects of TPA. Comparing two or more groups on a specific 
subject is a useful method for revealing group differences (43). According to Bergman et al. (53), a cluster analysis gives a thorough description of individual differences and allows for a translation of the results into properties characterising individuals. This gives a possibility to plan and offer individualized interventions based on the individual's difficulties and not only on the basis of the diagnosis.

In the present study, both parent-ratings and self-ratings were used. This gives a broad picture of children's functioning in everyday life. To obtain information from both children and their parents provides opportunities to both perspectives and could give important information concerning the child's health and well-being $(61,62)$.

The recruitment of children with ADHD had the purpose to find children that could benefit from the intervention described in Wennberg et al. (42). This makes the present study not representative for the proportion of DTM difficulties in all children with ADHD. A limitation is also the amount of dropouts of self-ratings of autonomy in children with ID. We don't know the reasons except for two children (didn't understand the questions and didn't want to answer). One explanation of the dropouts could be that the number of statements was too time consuming and demanding for the children since this self-rating was often done at the same time as the KaTid-measurement and a self-rating of DTM. Another explanation could be that the children with ID were unfamiliar with self-reporting questionnaires.

\section{Conclusions}

The present study supports the hypothesis that children with ADHD and ID have the same overall pattern of TPA but may have a delayed TPA, which affects their DTM and autonomy, and thereby also influence their participation in daily activities. This indicates that they could have specific problems in developing TPA in relation to their age, compared to TD children, and therefore need support in order to develop more advanced levels of TPA. However, not all children with ADHD or ID had specific difficulties with TPA, compared to TD children indicating a need to measure children's ability at each subscale in order to tailor a suitable intervention for every child in need of it. The results deepen and expand our knowledge of time deficits in children and adolescents with ADHD and ID in relation to the whole construct of time-processing ability; including time perception, time orientation and time management.

\section{Abbreviations}

ADHD Attention Deficit Hyperactivity Disorder

CAP Children and adolescent psychiatric clinic

DTM Daily time management

HAB Habilitation service

ID Intellectual disability

KaTid Kit for assessing time-processing ability

TD Typically developing [children]

TPA Time-processing ability

\section{Declarations}




\section{Ethics approval and consent to participate}

The ethical guidelines stipulated by the Helsinki Declaration (48) guided the research process. Ethical approval for this study was granted by the Regional Ethical Review Board in Linköping for the children with ADHD (D-nr. 2012/166-31) and for the children with ID and the TD children (D-nr. 2005/032).

Written informed consent was obtained from all parents and from the older children/adolescents in the study.

\section{Consent for publication}

Written informed consent for publication was obtained from each participant prior to data collection.

\section{Availability of data and materials}

The data that support the findings of this study are available from Linköping University but restrictions apply to the availability of these data, which were used under license for the current study. Data are thus not publicly available. Data are however available from the authors upon reasonable request and with permission of Linköping University.

\section{Competing interests}

The fifth author (GJ) developed the instrument KaTid-Youth measuring time-processing ability and the Time-Parent scale and sells the KaTid material and training courses to professionals. The other authors have no competing interests with respect to the research, authorship, and/or publication of this article.

\section{Funding}

Grants from the following research funds are acknowledged:

Vårdalstiftelsen Idéprovningsprojekt D-nr 98

Stiftelsen Sunnerdahls Handikappfon, nr 41/17

The Medical Research Council of Southeast Sweden [FORSS-305331 and FORSS-565411]

We also acknowledge Habilitering \& Hälsa, SLSO for financial support

The funding sources had no role in study design, the collection, analysis and interpretation of data, the writing of the report, or the decision to submit the paper for publication.

\section{Authors' contributions}

All authors, Birgitta Wennberg, Anette Kjellberg, Per A. Gustafsson, Lena Almqvist and Gunnel Janeslätt have contributed significantly to the manuscript and are in agreement on its content.

\section{Acknowledgements}

The authors would like to thank the children and their parents, as well as the staffs at the enrolled CAP/HAB clinics for their time and support during the data collection process. The occupational therapists collecting data and research assistant Annika Persson are gratefully acknowledged. Also thanks to Elisabet Walinder who helped the first author to compile comparative data and to professor Mats Granlund and professor Laura Korhonen for valuable comments. 


\section{References}

1. Davies DK, Stock SE, Wehmeyer ML. Enhancing independent time-management skills of individuals with mental retardation using a Palmtop personal computer. Mental retardation. 2002;40(5):358-65.

2. Janeslätt G, Granlund M, Kottorp A. Measurement of time processing ability and daily time management in children with disabilities. Disability and Health Journal. 2009;2:15-9.

3. Bylholt C. A Review of the Literature on the Acquisition and Development of Time Concepts in Children. CAEDHH Journal/La Revue ACESM. 1997;23(2-3):119-24.

4. Gillespie A, Best C, O'Neill B. Cognitive function and assistive technology for cognition: a systematic review. Journal of the International Neuropsychological Society : JINS. 2012;18(1):1-19.

5. World Health Organization. International classification of functioning, disability and health [Elektronisk resurs] children and youth version : ICF-CY. Geneva: World Health Organization; 2007 [cited 17 jan 31]. Available from: http://apps.who.int/iris/handle/10665/43737.

6. Janeslätt G. Validity in assessing time processing ability, test equating of KaTid-Child and KaTid-Youth. Child: care, health and development. 2012;38(3):371-8.

7. Janeslätt G, Granlund M, Kottorp A, Almqvist L. Patterns of Time Processing Ability in Children with and without Developmental Disabilities. Journal of Applied Research in Intellectual Disabilities. 2010;23(3):250-62.

8. Janeslätt G, Granlund M, Alderman I, Kottorp A. Development of a new assessment of time processing ability in children, using Rasch analysis. Child: care, health and development. 2008;34(6):771-80.

9. Barkley RA. Behavioral inhibition, sustained attention, and executive functions: constructing a unifying theory of ADHD. Psychological bulletin. 1997;121(1):65-94.

10. Diamond A. Executive functions. Annual review of psychology. 2013;64:135-68.

11. Cardol M, De Jong BA, Ward CD. On autonomy and participation in rehabilitation. Disability and rehabilitation. 2002;24(18):970-4; discussion 5-1004.

12. Chan RCK. Active participation and autonomy: An ultimate target for rehabilitation. Disability and Rehabilitation: An International, Multidisciplinary Journal. 2002;24(18):983-4.

13. Peny-Dahlstrand M, Krumlinde-Sundholm L, Gosman-Hedstrom G. Is autonomy related to the quality of performance of everyday activities in children with spina bifida? Disability and rehabilitation. 2012;34(6):51421.

14. Rosenberg L. The Associations Between Executive Functions' Capacities, Performance Process Skills, and Dimensions of Participation in Activities of Daily Life Among Children of Elementary School Age. Applied neuropsychology Child. 2015;4(3):148-56.

15. Imms C, Granlund M, Wilson PH, Steenbergen B, Rosenbaum PL, Gordon AM. Participation, both a means and an end: a conceptual analysis of processes and outcomes in childhood disability. Developmental medicine and child neurology. 2016.

16. Almqvist L, Granlund M. Participation in school environment of children and youth with disabilities: a personoriented approach. Scandinavian journal of psychology. 2005;46(3):305-14.

17. Eriksson L, Granlund M. Perceived participation. A comparison of students with disabilities and students without disabilities. Scandinavian Journal of Disability Research. 2004;6(3):206-24.

18. Lachapelle Y, Wehmeyer ML, Haelewyck MC, Courbois Y, Keith KD, Schalock R, et al. The relationship between quality of life and self-determination: an international study. Journal of intellectual disability research : JIDR. 
2005;49(Pt 10):740-4.

19. Willcutt EG, Doyle AE, Nigg JT, Faraone SV, Pennington BF. Validity of the Executive Function Theory of Attention-Deficit/Hyperactivity Disorder: A Meta-Analytic Review. Biological psychiatry. 2005;57(11):1336-46.

20. Faraone SV, Asherson P, Banaschewski T, Biederman J, Buitelaar JK, Ramos-Quiroga JA, et al. Attentiondeficit/hyperactivity disorder. Nature reviews Disease primers. 2015;1:15020.

21. Shaw P, Malek M, Watson B, Sharp W, Evans A, Greenstein D. Development of cortical surface area and gyrification in attention-deficit/hyperactivity disorder. Biological psychiatry. 2012;72(3):191-7.

22. Smith A, Taylor E, Rogers JW, Newman S, Rubia K. Evidence for a pure time perception deficit in children with ADHD. Journal of child psychology and psychiatry, and allied disciplines. 2002;43(4):529-42.

23. Rubia K, Halari R, Christakou A, Taylor E. Impulsiveness as a timing disturbance: neurocognitive abnormalities in attention-deficit hyperactivity disorder during temporal processes and normalization with methylphenidate. Philosophical transactions of the Royal Society of London Series B, Biological sciences. 2009;364(1525):191931.

24. Toplak ME, Dockstader C, Tannock R. Temporal information processing in ADHD: findings to date and new methods. Journal of neuroscience methods. 2006;151(1):15-29.

25. Falter CM, Noreika V. Interval timing deficits and abnormal cognitive development. Frontiers in integrative neuroscience. 2011;5:26.

26. Noreika V, Falter CM, Rubia K. Timing deficits in attention-deficit/hyperactivity disorder (ADHD): evidence from neurocognitive and neuroimaging studies. Neuropsychologia. 2013;51(2):235-66.

27. Walg M, Hapfelmeier G, El-Wahsch D, Prior H. The faster internal clock in ADHD is related to lower processing speed: WISC-IV profile analyses and time estimation tasks facilitate the distinction between real ADHD and pseudo-ADHD. European child \& adolescent psychiatry. 2017;26(10):1177-86.

28. Mioni G, Santon S, Stablum F, Cornoldi C. Time-based prospective memory difficulties in children with ADHD and the role of time perception and working memory. Child Neuropsychology. 2016:1-21.

29. Abikoff H, Gallagher R, Wells KC, Murray DW, Huang L, Lu F, et al. Remediating organizational functioning in children with ADHD: immediate and long-term effects from a randomized controlled trial. Journal of consulting and clinical psychology. 2013;81(1):113-28.

30. Lindblad I, Svensson L, Landgren M, Nasic S, Tideman E, Gillberg C, et al. Mild intellectual disability and ADHD; a comparative study of school age children's adaptive abilities. Acta paediatrica (Oslo, Norway : 1992). 2013;102(10):1027-31.

31. Kylén G, af Sandeberg I. Begåvning och begåvningshandikapp. Stockholm :: ALA ; 1986.

32. Owen AL, Wilson RR. Unlocking the riddle of time in learning disability. Journal of intellectual disabilities : JOID. 2006;10(1):9-17.

33. American Association on Intellectual and Developmental Disabilities. Intellectual disability : definition, classification, and systems of supports. Washington, DC: American Association on Intellectual and Developmental Disabilities; 2010. 259 p.

34. Levén A, Lyxell B, Andersson J, Danielsson H, Rönnberg J. Prospective Memory, Working Memory, Retrospective Memory and Self-Rated Memory Performance in Persons with Intellectual Disability. Scandinavian Journal of Disability Research. 2008;10(3):147-65.

35. Arvidsson G, Jonsson $\mathrm{H}$. The impact of time aids on independence and autonomy in adults with developmental disabilities. Occupational therapy international. 2006;13(3):160-75.

Page $17 / 23$ 
36. Janeslätt G, Kottorp A, Granlund M. Evaluating intervention using time aids in children with disabilities. Scandinavian journal of occupational therapy. 2014;21(3):181-90.

37. Wennberg B, Kjellberg A. Participation when using cognitive assistive devices--from the perspective of people with intellectual disabilities. Occupational therapy international. 2010;17(4):168-76.

38. Granlund M, Bond A, Lindström E, Wennberg B. Assistive Technology and Persons with Developmental Disabilities. Technology and Disability. 1995; 4(3-4):205-14.

39. Janeslätt G, Wallin Ahlström S, Granlund M. Intervention in time-processing ability, daily time management and autonomy in children with intellectual disabilities aged 10-17 years - A cluster randomised trial. Australian Occupatinal Therapy Journal. 2018.

40. Eriksson L, Welander J, Granlund M. Participation in Everyday School Activities For Children With and Without Disabilities. Journal of Developmental and Physical Disabilities. 2007;19(5):485-502.

41. Walg M, Oepen J, Prior H. Adjustment of Time Perception in the Range of Seconds and Milliseconds: The Nature of Time-Processing Alterations in Children With ADHD. Journal of attention disorders. 2015;19(9):75563.

42. Swedish agency for health technology assessment and assessment of social services. ADHD : diagnostik och behandling, vårdens organisation och patientens delaktighet : en systematisk litteraturöversikt [ADHD diagnostics and treatment, organization of the health care and patient involvement: A systematic literature review] Stockholm: Statens beredning för medicinsk utvärdering (SBU); 2013 [cited 2017 jan 31]. Available from: http://www.sbu.se/217.

43. Polit DF, Beck CT. Nursing research : generating and assessing evidence for nursing practice. Philadelphia: Wolters Kluwer Health/Lippincott Williams \& Wilkins; 2008.

44. Kadesjo B, Janols LO, Korkman M, Mickelsson K, Strand G, Trillingsgaard A, et al. The FTF (Five to Fifteen): the development of a parent questionnaire for the assessment of ADHD and comorbid conditions. European child \& adolescent psychiatry. 2004;13 Suppl 3:3-13.

45. Wennberg B, Janeslätt G, Kjellberg A, Gustafsson PA. Effectiveness of time-related interventions in children with ADHD aged 9-15 years: a randomized controlled study. European child \& adolescent psychiatry. 2018;27(3):32942.

46. Wehmeyer ML, Kelchner K. Measuring the Autonomy of Adolescents and Adults with Mental Retardation : A Self-Report Form of the Autonomous Functioning Checklist. Career Development for Exceptional Individuals. 1995;18(1):3-20.

47. Sigafoos AD, Feinstein $C B$, Damond $M$, Reiss $D$. The measurement of behavioral autonomy in adolescence: the autonomous functioning checklist. Adolescent psychiatry. 1988;15:432-62.

48. World Medical Association of Helsinki. World Medical Association declaration of Helsinki : ethical principles for medical research involving human subjects. Seoul Guildford: Canary Publications; 2008.

49. Corcoran K, Fischer J. Measures for clinical practice : a sourcebook. Vol. 1, Couples, families and children. New York: Free Press; 2000.

50. Wright DB, London K, Field AP. Using Bootstrap Estimation and the Plug-in Principle for Clinical Psychology Data. Journal of Experimental Psychopathology. 2011;2(2):252-70.

51. Cohen J. Statistical power analysis for the behavioral sciences. Hillsdale: L. Erlbaum Associates; 1988.

52. Bergman \& El-Khouri. SLEIPNER: A statistical package for pattern-oriented anayses (Version 2.1)[Computer software]. 2002. 
53. Bergman LR, Magnusson D, El-Khouri BM. Studying individual development in an interindividual context: A person-oriented approach. Mahwah: NJ: Erlbaum Associates, Inc.; 2003.

54. Field A. Discovering statistics using IBM SPSS statistics : and sex and drugs and rock 'n' roll. Los Angeles;; Sage; 2013.

55. Langberg JM, Epstein JN, Graham AJ. Organizational-skills interventions in the treatment of ADHD. Expert review of neurotherapeutics. 2008;8(10):1549-61.

56. Mechling LC. Review of twenty-first century portable electronic devices for persons with moderate intellectual disabilities and autism spectrum disorders. Education and Training in Autism and Developmental Disabilities. 2011;46(4):479-98.

57. Wehmeyer ML, Palmer SB, Smith SJ, Davies DK, Stock S. The Efficacy of Technology Use by People with Intellectual Disability: A Single-Subject Design Meta-Analysis. Journal of Special Education Technology. 2008;23(3):21-30.

58. Koyama T, Wang HT. Use of activity schedule to promote independent performance of individuals with autism and other intellectual disabilities: a review. Res Dev Disabil. 2011;32(6):2235-42.

59. Bikic A, Reichow B, McCauley SA, Ibrahim K, Sukhodolsky DG. Meta-analysis of organizational skills interventions for children and adolescents with Attention-Deficit/Hyperactivity Disorder. Clin Psychol Rev. 2017;52:108-23.

60. Booster GD, Dupaul GJ, Eiraldi R, Power TJ. Functional impairments in children with ADHD: unique effects of age and comorbid status. Journal of attention disorders. 2012;16(3):179-89.

61. Hemmingsson H, Olafsdottir LB, Egilson ST. Agreements and disagreements between children and their parents in health-related assessments. Disability and rehabilitation. 2016:1-14.

62. Klassen AF, Miller A, Fine S. Agreement between parent and child report of quality of life in children with attention-deficit/hyperactivity disorder. Child: care, health and development. 2006;32(4):397-406.

\section{Tables}




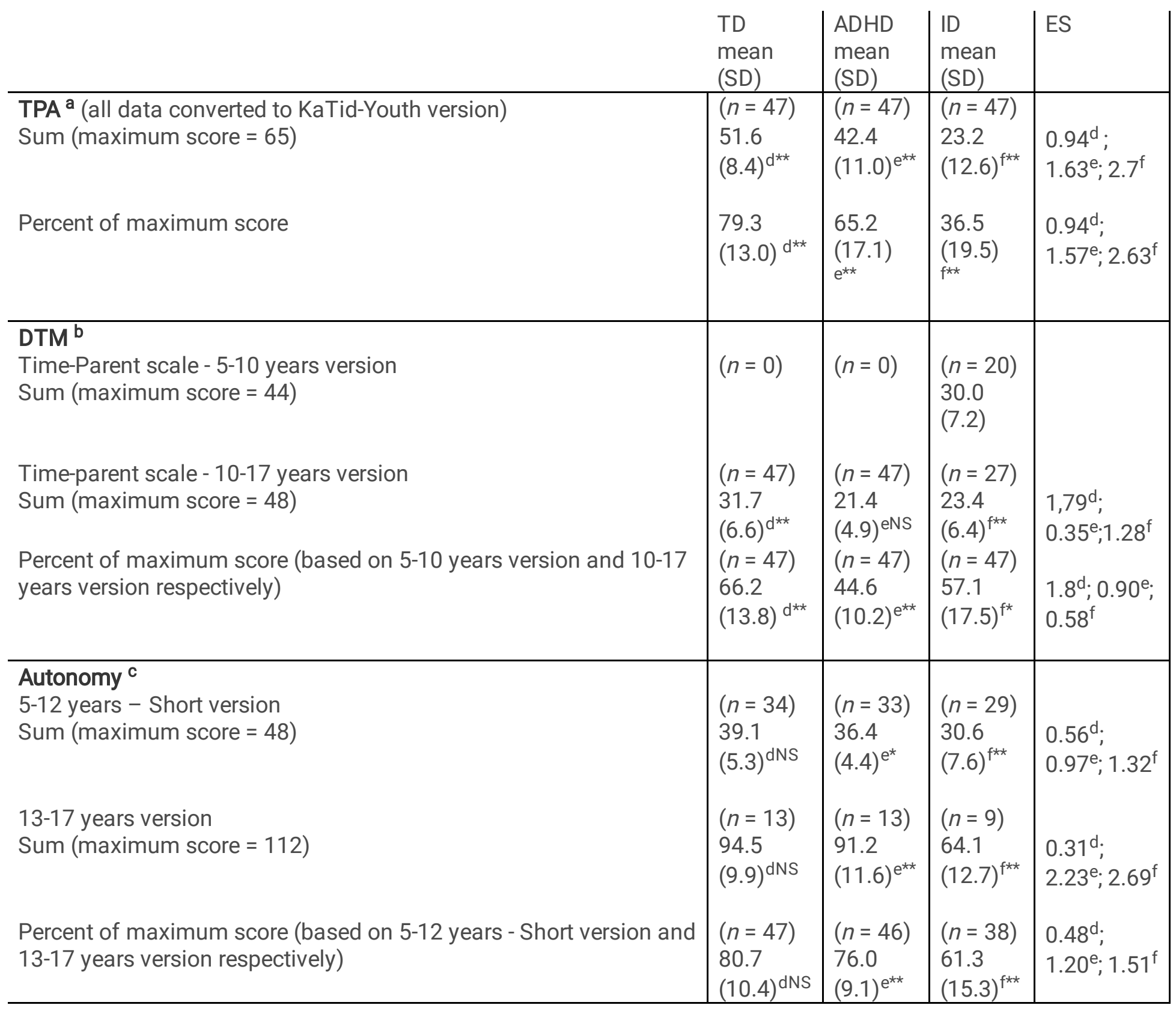

Group differences in scores and percentage of maximum scores for time-processing ability (TPA) a, daily time management (DTM) ${ }^{\mathrm{b}}$ and self-rated Autonomy ${ }^{\mathrm{c}}$ among typically developing (TD) children, children with ADHD and children with ID.

$\mathrm{d}=\mathrm{TD}$ compared to ADHD

e $=$ ADHD compared to ID

$\mathrm{f}=\mathrm{TD}$ compared to ID

$N S=$ non-significant, ${ }^{*}=p<.05, * *=p<.01$

$\mathrm{ES}=$ Effect size using Cohen's $d$ 


\begin{tabular}{|c|c|c|c|c|c|}
\hline & Cluster A & Cluster B & Cluster C & Cluster D & All clusters \\
\hline Homogeneity & .709 & .706 & .451 & 1.131 & \\
\hline$n(\%)$ & $79(56.8)$ & $46(33.2)$ & $7(5)$ & $7(5)$ & $139(100)$ \\
\hline \multicolumn{6}{|l|}{$\operatorname{Sex} n(\%)$} \\
\hline Girls & $25(32)$ & $15(33)$ & $1(14)$ & $3(43)$ & $44(32)$ \\
\hline Boys & $54(68)$ & $31(67)$ & $6(86)$ & $4(57)$ & $95(68)$ \\
\hline \multicolumn{6}{|l|}{ Diagnosis/no diagnosis $n(\%)$} \\
\hline TD & $37(78.7)$ & $10(21.3)$ & & & $47(100)$ \\
\hline ADHD & $33(70.2)$ & $12(25.5)$ & $2(4.3)$ & & $47(100)$ \\
\hline ID & $9(20.0)$ & $24(53.3)$ & $5(11.1)$ & $7(15.6)$ & $45(100)$ \\
\hline \multicolumn{6}{|l|}{ Age $M$ (SD; min-max) } \\
\hline All children in the cluster & $\begin{array}{l}11.8 \\
(1.92 ; \\
8.72-15.73)\end{array}$ & $\begin{array}{l}10.9 \\
(1.66 ; \\
8.62-15.14)\end{array}$ & $\begin{array}{l}11.1 \\
(1.42 ; \\
9.44-13.50)\end{array}$ & $\begin{array}{l}12.5 \\
(2.24 ; \\
9.66-15.58)\end{array}$ & $\begin{array}{l}11.5 \\
(1.88 ; \\
8.62-15.73)\end{array}$ \\
\hline TD & $\begin{array}{l}12.0 \\
(1.86 ; \\
9.00-15.64)\end{array}$ & $\begin{array}{l}9.8 \\
(.73 \\
9.11-11.32)\end{array}$ & - & - & \\
\hline ADHD & $\begin{array}{l}11.7 \\
(1.99 ; \\
8.72-15.73)\end{array}$ & $\begin{array}{l}11.2 \\
(1.79 ; \\
8.62-15.14)\end{array}$ & $\begin{array}{l}9.7 \\
(.30 ; \\
9.44-9.86)\end{array}$ & - & \\
\hline ID & $\begin{array}{l}11.5 \\
(2.07 \\
9.17-15.08)\end{array}$ & $\begin{array}{l}11.2 \\
(1.74 ; \\
8.66-14.92)\end{array}$ & $\begin{array}{l}11.6 \\
(1.28 \\
10.42-13.50)\end{array}$ & $\begin{array}{l}12.5 \\
(2.25 \\
9.66-15.58)\end{array}$ & \\
\hline \multicolumn{6}{|l|}{ TPA $^{\mathrm{a}} M$} \\
\hline $\begin{array}{l}\text { TPA Total score, } \\
(S D)\end{array}$ & $82(8.42)$ & $58(8.85)$ & $39(4.15)$ & $24(8.71)$ & \\
\hline Time perception & 88 & 77 & 86 & 48 & \\
\hline $\begin{array}{l}\text { Time orientation/ } \\
\text { concepts }\end{array}$ & 89 & 76 & 56 & 44 & \\
\hline $\begin{array}{l}\text { Time orientation/ } \\
\text { objective time }\end{array}$ & 78 & 48 & 16 & 11 & \\
\hline Time management & 71 & 32 & 6 & 2 & \\
\hline DTM $^{\mathrm{b}} M(S D)$ & $57(16.3)$ & $56(16.7)$ & $60(14.2)$ & $40(14.4)$ & \\
\hline Autonomy ${ }^{\mathrm{c}} M(\mathrm{SD})$ & $78(10.7)$ & $71(12.6)$ & $60(16.1)$ & $42(9.1)$ & \\
\hline
\end{tabular}

Time-processing ability (TPA) ${ }^{\mathrm{a}}$ as percentage of total score and subscale scores, Daily time management (DTM) ${ }^{\mathrm{b}}$ as percentage of total score and self-rated Autonomy ${ }^{\mathrm{C}}$ as percentage of total score, in the clusters.

\section{Figures}




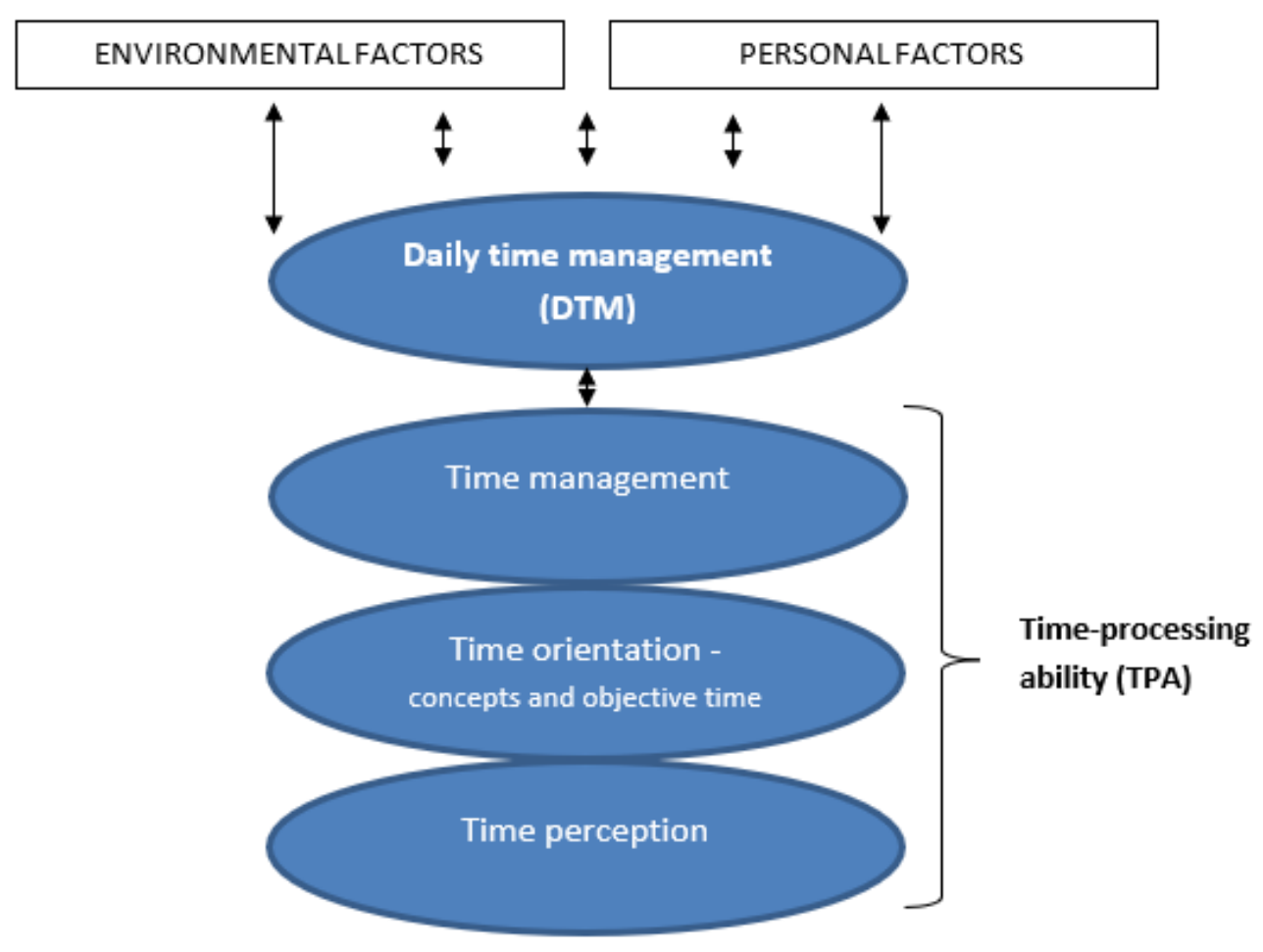

\section{Figure 1}

Description of the relation between Daily time management (DTM) and Time-processing ability (TPA), Environmental factors, and Person factors. Legend: Modified from Time for time (Janeslätt, 2009). 


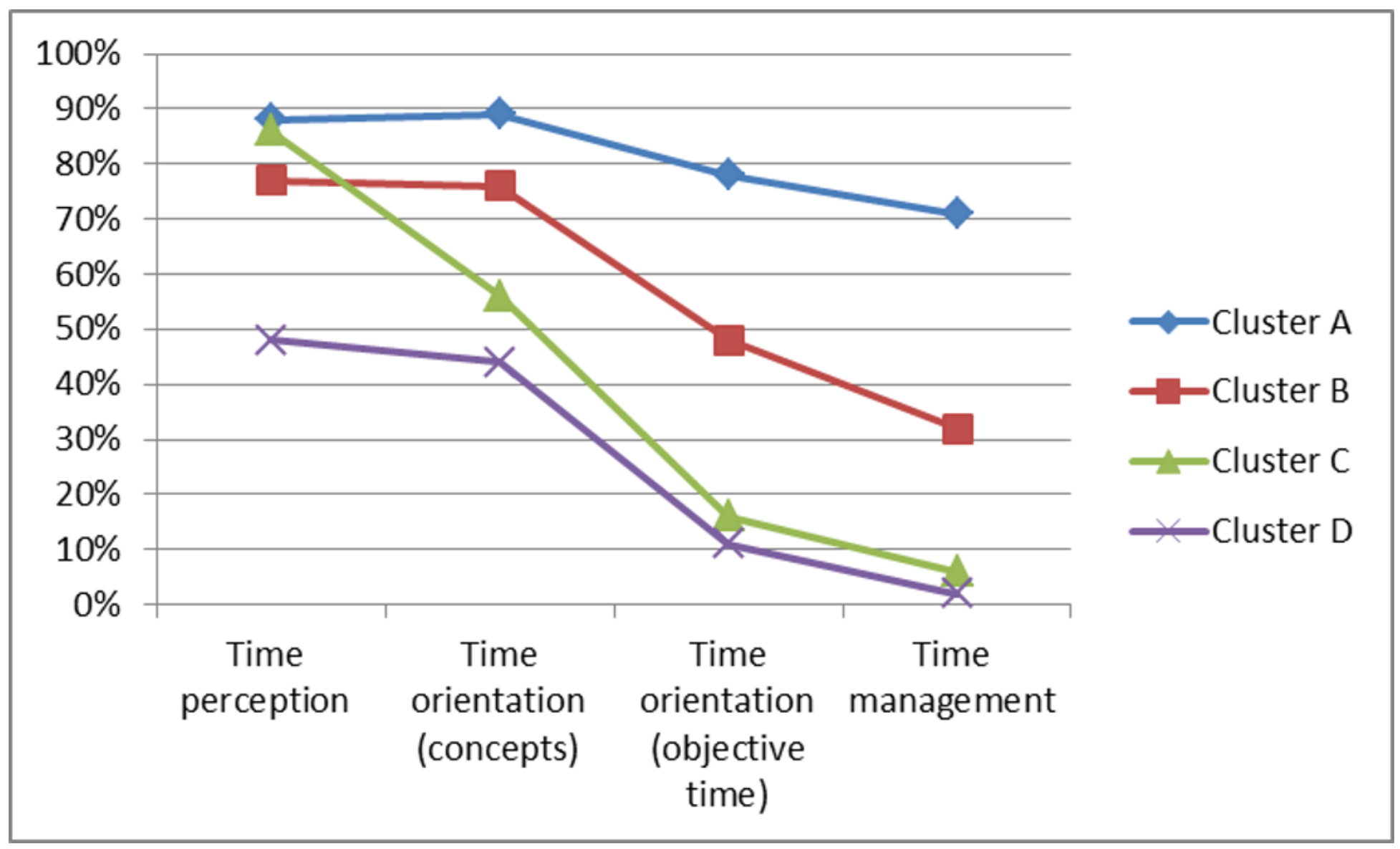

\section{Figure 2}

Time-processing ability in percent of maximum scores in each subscale of KaTid for the clusters. Legend: Cluster A Skilled in all levels of TPA ( $n=79$, TD 47\%, ADHD 42\%, IFD11\%) Cluster B - Skilled in time perception and time orientation/concepts ( $n=46$, TD 26\%, ADHD 22\%, ID 52\%) Cluster C - Skilled only in time perception ( $n=7$, TD 0\%, ADHD 29\%, ID 71\%) Cluster D - Difficulties at all levels of TPA ( $n=77$, TD 0\%, ADHD 0\%, ID 100\%) 